\title{
An investigation of the structure of expectancy-value attitude and its implications
}

\section{Youjae YI*}

The purpose of this study was to propose a new way of representing the structure of expectancy-value attitude and to investigate the implications of its representation. Expectancyvalue attitude was represented as a cognitive network of vertically and horizontally interconnected judgements about product attributes, using a structural equation modeling methodology. Results suggested that a structural representation of expectancy-value attitude gave a satisfactory fit to the data. Two implications of this representation were also tested for: (1) understanding the effects of expectancy-value attitude on intentions, and (2) predicting the dynamics of belief change.

The structural representation of expectancy-value attitude did not improve the prediction of intentions. The observed effects of expectancy-value attitude on intentions did not differ between the traditional and proposed structural representations. However, the structural representation was useful for understanding belief change. Since expectancy-value attitude existed as a network of interconnected beliefs, an ad effect on one belief was hypothesized to bring about changes in other related beliefs. As hypothesized, advertisements mentioning one belief changed other beliefs that were connected with the mentioned belief.

\section{Introduction}

The expectancy-value (EV) model posits that an individual's attitude toward an act (Aact) is a function of a belief-evaluation composite (hereafter referred to as EV attitude). ${ }^{1}$ The traditional model represents EV attitude as a single value by using the sum of belief-

\footnotetext{
* Youjae $\mathrm{Yi}$ is an Assistant Professor of Marketing at the School of Business Administration, The University of Michigan, Ann Arbor, MI 48109-1234, U.S.A.
}

Intern. J. of Research in Marketing 6 (1989) 71-83

North-Holland times-evaluation products (Ajzen and Fishbein, 1980; Fishbein and Ajzen, 1975). This representation implicitly assumes that individual reactions to product attributes are independent. However, a number of developments challenge this assumption. For example, Beckwith and Lehmann (1973) have found that beliefs about certain attributes are often correlated in product evaluations. Bagozzi (1982) criticizes the assumption that product attributes are independent and suggests several conditions under which non-independence of attributes might be expected. These findings suggest that the traditional representation of EV attitude based on such an assumption may be less than satisfactory.

Several researchers have thus extended the traditional EV model so as to capture such interrelationships among attributes and suggested a structural representation of EV attitude (see, e.g., Bagozzi, 1985; Burnkrant and Page, 1988). This suggestion is consistent with the findings in cognitive psychology that human judgements are stored in memory in the

\footnotetext{
Although the term 'EV attitude' has been used to refer to the belief-evaluation composite in marketing and psychology (see, e.g., Bagozzi, 1981. 1985; Bumkrant and Page, 1988), 'cognitive structure' has also been used by many researchers (see, e.g., Lutz, 1977). EV attitude should be distinguished from attitude toward the act (A-act). EV attitude provides an indirect, cognitive measure of attitudinal responses through assessment of a person's beliefs and evaluations about various consequences of an act, whereas A-act is a direct, affective measure of a person's feelings toward an overall act (see Bagozzi (1981) for more discussion of the distinction between the two constructs).
} 
form of a network (Anderson, 1983; Collins and Loftus, 1975). Indeed, many studies have shown that EV attitude can be represented as a multidimensional structure (Bagozzi, 1981, 1983; Shimp and Kavas, 1984; Oliver and Bearden, 1985). Nevertheless, they have not fully explored why the structure of EV attitude is relevant to marketing theory and practice and it is unclear at this point what are the benefits of employing a structural form of $\mathrm{EV}$ attitude.

The purpose of the present study is to extend extant research on EV attitude further and to examine the implications of representing EV attitude in a structural form for understanding attitude formation and change. It is hypothesized that probing the structure of EV attitude can enhance (1) prediction of intentions, and (2) understanding of attitude change processes. Specifically, this study investigates how representations of $\mathrm{EV}$ attitude affect the observed relationship between EV attitude and intentions and what insights the structure of $\mathrm{EV}$ attitude provides for predicting indirect effects of advertising on beliefs.

\section{Existing models of EV attitude}

We can identify three models of EV attitude in previous research: the traditional EV model, the modified unidimensional EV model, and the multidimensional EV model. These models are illustrated in Fig. 1.

The traditional EV model has dominated past studies (Fishbein and Ajzen, 1977; Lutz, 1977). As Fig, 1A indicates, this model defines $\mathrm{EV}$ attitude to be $\sum B_{i} a_{i}$, a point representation of aggregated beliefs about and evaluations of the outcomes of an act. Individual beliefs or evaluations lose their meaning to a certain extent, since different beliefs and evaluations can give the same overall sum. Measurement errors are not explicitly modeled, and reliability cannot be assessed (except for test-retest reliability). Further-
A. Traditional EV model

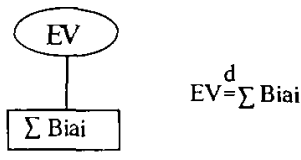

B. Modified unidimensional EV model

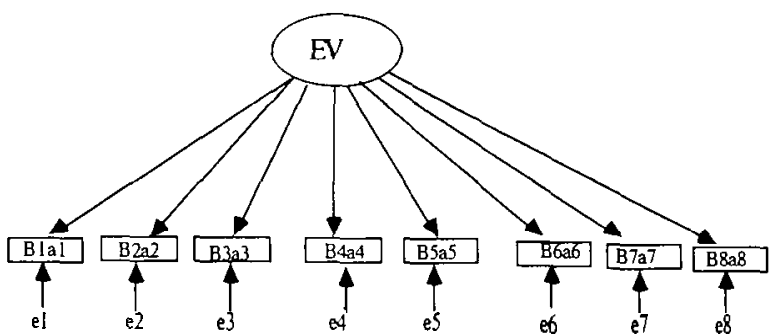

C. Multidimensional EV Model

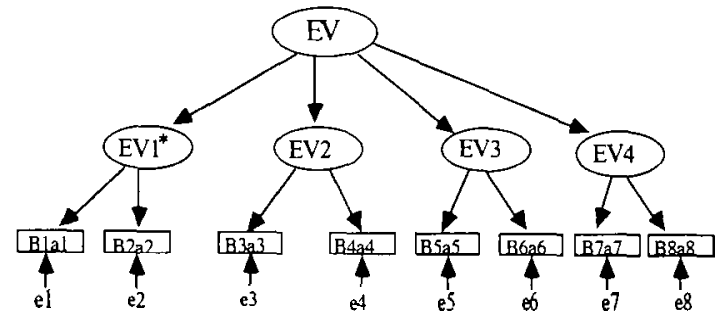

Fig. 1. An illustration of the three types of existing EV models. (The $\mathrm{EV}_{i}$ 's, I $=1.2$, 3, 4, indicate subdimensions of $\mathrm{EV}$ attitude.)

more, it is difficult to assess the construct validity of the traditional EV model, and most validations have been done through examination of only predictive validity (Wilkie and Pessemier, 1973).

The modified unidimensional EV model, suggested by Bagozzi (1982, 1985), regards $\mathrm{EV}$ attitude as unidimensional, but it retains the identity of individual belief-times-evaluation products. EV attitude is treated as a latent variable, and all belief-times-evaluation products are treated as its indicators. As shown in Fig. 1B, each expectancy-value measure is a function of measurement error as well as a latent variable, where we have assumed that there are eight attributes for the sake of illustration. In this model, various forms of reliability can be computed, and construct validity can be assessed. 
The multidimensional EV model treats each expectancy-value measure as a separate indicator of a latent variable, but it permits the dimensionality so that different subsets of belief-times-evaluation products serve as indicators of subdimensions (EV,) (Bagozzi, 1982, 1983). In Fig. 1C, for example, the first two attributes are measures of the same dimension. Each dimension $(\mathrm{EV}$,$) is also con-$ strued as an indicator of a higher-order latent variable (EV). This model incorporates the interdependence information by using a hierarchical structure, but it permits only one type of interdependence (i.e., measures of the same concept). It is argued herein that this model is not complete since it does not consider other types of interdependence which will be discussed in the next section.

\section{3. $\mathrm{H}$ ypotheses}

\subsection{The interdependence EV model}

A basic premise of this study that there may be interdependences (or perceived corre- lations) among product attributes. Let us examine several types of interdependence among attributes, which are illustrated in Fig. 2.

The first type of interdependent attributes consists of causally related attributes. For example, if consumers believe that reliability of a car will reduce maintenance costs, 'reliability' and 'maintenance costs' will be negatively associated. A second type comprises semantically correlated attributes as measures of the same concept. This type provides a basis for representing the vertical structure with a hierarchy of attributes, which may occur because people abstract information about general attributes on the basis of several more concrete attributes. Suppose attributes 3 and 4 in Fig. 2 are 'gas mileage (mpg)' and 'fuel economy'. Then, these attributes will be correlated since they are likely to mean the same thing to the consumer.

Another type of interdependence is ecological correlation. In this case, attributes happen to be correlated by environmental characteristics. For example, 'European' and "prestigious" are likely to be associated with

Type I: Interdependence by a causal relation

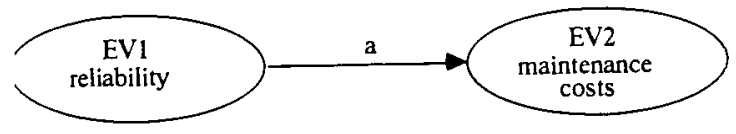

Type II: Interdependence by measuring the same concept

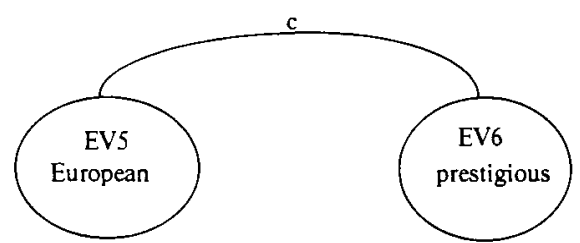

Type IV: Interdependence by sharing a common antecedent
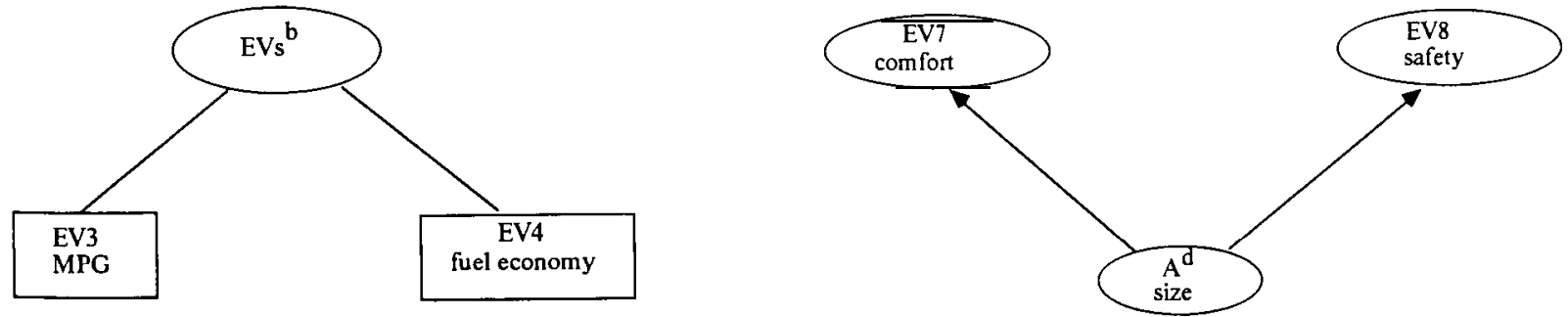

Fig. 2. An illustration of several types of interdependence. a: the arrow indicates a causal relationship; b: $E V_{s}$ indicates a subdimension of EV attitude; c: the curved line indicates an ecological association; d: A indicates a common antecedent. 
each other, since many European cars (e.g., Rolls Royce, Mercedes, Volvo) have been prestigious over the years. Still another type of interdependence comes from sharing a common antecedent. For instance, knowledge about the size of a car might influence one's perceptions of overall comfort as well as safety. In such a case, perceptions of 'overall comfort' and 'safety' are interdependent because of their dependence on a common antecedent.

It is asserted in this paper that a representation of $\mathrm{EV}$ attitude should take into account interdependence among product attributes. 2 An interdependence EV model will be hereafter used to refer to a model that represents EV attitude as a network-like structure by explicitly incorporating such interdependence. In this model, EV judgements are vertically interrelated via a hierarchy of attributes and horizontally connected at the same level of abstraction. But how valid is this representation of $\mathrm{EV}$ attitude?

2 An anonymous reviewer argued that since the EV model (implicitly) assumes independent beliefs, one should make sure that the beliefs are independent prior to the application of the EV model. This could be achieved by either choosing only independent beliefs or extracting orthogonal dimensions (e.g., via factor analysis) from the set of originally elicited beliefs. However, the original set of salient beliefs was used in this study for several reasons. First, the orthogonalization of beliefs is seldom suggested explicitly or used in practice by EV reachers (see, e.g., Ajzen and Fishbein, 1980; Lutz, 1977). For example, such a procedure is not mentioned when the steps of eliciting salient beliefs are presented in detail (Ajzen and Fishbein, 1980, pp. 260-263). Second, such a procedure loses information about consumers' actual judgements and evaluations. Even if one can derive some independent beliefs, it is unlikely that consumers will actually use these dimensions in product evaluations. It seems more informative to use the original beliefs generated by consumers than the derived beliefs extracted by researchers. Third, previous researchers have demonstrated that interrelated beliefs can be modeled within the expectancy-value framework (Bagozzi, 1982, 1985; Bumkrant and Page, 1988; Shimp and Kavas, 1984). Finally, the purpose of the present study is to examine the benefits of modeling explicitly the interrelations among beliefs. That is, the present study models explicitly the processes underlying attribute redundancy, rather than artificially removing correlations among attributes as would be suggested in the traditional approach.
The interdependence EV model looks deep into EV attitude and exposes the underlying substructures. Strictly speaking, the traditional EV model is not a cognitive structure, since it maps onto a single value and fails to reveal the structure of attitude. The interdependence $\mathrm{EV}$ model is indeed a cognitive structure in that it contains information about substructures and their relationships. Since the interdependence EV model provides a more explicit representation of the cognitive structure, it should achieve higher convergent validity than existing EV models. Since it is difficult to assess the convergent validity of the traditional EV model which uses a single measure, no direct comparison is made with the traditional EV model.

Hypothesis 1. The interdependence EV model will achieve higher convergent validity than the modified unidimensional $E V$ or multidimensional E V models.

\subsection{Implications of $E V$ attitude representation}

What are the implications of representing EV attitude as a cognitive network? This question is addressed in two aspects: (1) understanding the effects of EV attitude on intentions, and (2) predicting dynamics of belief change.

\section{Effects of EV attitude on intentions.}

Existing findings concerning the effects of EV attitude on intentions are mixed. Traditional EV researchers have viewed that EV attitude influences intentions only indirectly though its effects on affect toward an act (A-act), which has been supported in many studies (Fishbein and Ajzen, 1975; Lutz, 1977). An alternative view is that affect does not completely mediate the effects of EV attitude on intentions; this may occur because cognitive elements of EV attitude (e.g., beliefs) are frequently too complex, and the 
cognitive processing capacities too imperfect for $\mathrm{EV}$ attitude to be completely processed into affect (Schlegel and DiTecco, 1982; Liska, 1984). This view suggests that EV attitude can have direct, unmediated by A-act, effects on intentions; the direct path has been found by some researchers (Bagozzi, 1982).

How can one explain such conflicting findings? A possible answer comes from considering representations of EV attitude. Since many traditional researchers have used a single value representation of $\mathrm{EV}$ attitude, one can attribute the insignificant direct effect found by them to the coarse-grained representation of EV attitude. Insignificant direct effects might have been an artifact of using a point representation of $\mathrm{EV}$ attitude which in fact exists as a cognitive network, since treating measures of multidimensional constructs as if they are unidimensional representations can lead to invalid predictions (Bagozzi, 1983).

The traditional EV model also assumes that measurement error is negligible. To the extent this assumption is violated, the observed relationship between EV attitude and intentions will be generally attenuated. As a result, a direct path from $\mathrm{EV}$ attitude to intentions is less likely to be observed under a traditional EV model. In contrast, since the interdependence EV model provides a richer and more reliable description of EV attitude by considering structures and measurement errors, it should reveal the direct path from EV attitude to $\mathrm{BI}$ better than the traditional $\mathrm{EV}$ model. ${ }^{3}$

$\mathbf{H}$ ypothesis 2. The direct effect of EV attitude on intentions is more likely to be observed under the interdependence EV model than under the traditional EV model.

3 A reviewer suggested that the traditional EV model will perform well (without the need for a direct link to $\mathrm{BI}$ ) if $\mathrm{EV}$ elements are measured and modeled adequately. This hypothesis can be tested in future research.
Belief change

What does the structure of EV attitude imply for belief change? Since EV attitude is an interconnected network, a change in one element would induce changes in other elements. Theoretical support comes form the spreading-activation theory, which posits that when one concept is activated, the activation is likely to spread to other concepts via associative linkages (Collins and Loftus, 1975). When ad recipients are exposed to an ad mentioning a certain attribute, other connected attributes are likely to be activated as well through the associative network. That is, product attribute beliefs are likely to become accessible according to the manner in which attributes are organized in EV attitude. As a result, people are likely to make inferences about these interdependent attributes (made accessible through spreading activation) and change beliefs about these attributes.

$\mathbf{H}$ ypothesis 3. An ad mentioning one belief will induce changes in other beliefs that are interdependent with the mentioned belief:

\section{M ethod}

\section{I. Procedure}

The subjects were 120 business school students and staff at a West Coast university in the United States. The test product was the Hyundai Excel, an imported car from South Korea. A focus group interview was administered to twenty students (who were not included in the main study) to identify salient beliefs about the product. Eight beliefs that were mentioned by at least $30 \%$ of the individuals were selected as salient beliefs underlying attitudes, i.e., dependability, riding comfort, repair costs, sportiness, ease of maintenance, roominess, style, and durability. 
In the main study, each subject was given an envelope with four booklets and told to complete the booklets in order. In the first booklet, subjects were asked for general background information such as their attitude, experience and knowledge about cars in general. They were then asked about pre-exposure attitudinal responses, including beliefs, evaluations, attitude toward the act, subjective norm, and behavioral intention. Next, they filled out questions on several personality variables, which served as filler tasks between the pre-test and post-test to reduce memory effects.

In the next booklet, each subject saw an ad with messages emphasizing either dependability or repair costs. Each subject was told a disguised purpose of the study consisting of the evaluation of print ads in pre-production form. In the final booklet, subjects filled out a post-exposure questionnaire on attitudinal responses, including expectancy-value measures.

\subsection{Data collection instruments}

To assess beliefs $\left(B_{i}\right)$ about the consequences of buying the Hyundai Excel, subjects were asked to estimate the probability that each consequence would occur on 11point scales, ranging from 'very unlikely' (0) to 'very likely' (10). The evaluative component $(a$, ) corresponding to the salient beliefs was measured by asking subjects to evaluate the consequence of each belief item on 11point bipolar scales ranging from 'very bad' $(-5)$ to 'very good' $(+5)$.

Affect toward the act (A-act) was measured on four 7-point bipolar scales: $\mathrm{good} / \mathrm{bad}$, pleasant/unpleasant, harmful/beneficial, and foolish/wise. Subjective norm was measured with the standard "Most people who are important to me think I should/ should not buy" item. Behavioral intentions (BI) to buy the Hyundai Excel were measured on three-item scales following the question: "What are your chances of buying the Hyundai Excel the next time you need to purchase a car?". The responses were measured on 1 1-point scales anchored with unlikely/ likely, improbable/ probable, and impossible/ possible.

\subsection{Reducing the threats to internal validity}

A number of precautions were taken to protect the validity of the results throughout the research. First, several steps were taken to reduce any demand characteristics. The purpose of the study was disguised with a plausible cover story, and several filler items were used throughout the questionnaire. The data were also collected with separate booklets in stages, and subjects were not allowed to refer back to their earlier responses. The post-experimental inquiry revealed that no subjects guessed the real purpose of the study. Second, multiple-item measures were taken for most key variables, which allowed us to correct for measurement errors in assessing the theoretical relationships. Third, half of the multiple response questions were reversed in their direction to eliminate any yea- or nay-saying biases from the responses. Fourth, the effect of subjective norm, another antecedent of intentions, was controlled for in assessing the effect of EV attitude on intentions (Ajzen and Fishbein, 1980).

\section{Results}

The hypotheses implied by the interdependence EV model were tested using a structural equation modeling framework. The LISREL VI was used to analyze the data, and the maximum likelihood fitting function was used to estimate the model (Jöreskog and Sörbom, 1984). 


\subsection{Interdependence $E V$ model}

It was hypothesized that EV attitude has an interdependent structure in that subsets of these attributes form distinct dimensions and some dimensions might be associated with others. Preliminary analysis suggested that there may be four dimensions underlying the attribute set chosen for this study: (1) reliability (i.e., durability and dependability), (2) maintenance costs (i.e., ease of maintenance and repair costs), (3) comfort (i.e., riding comfort and roominess), and (4) appearance (i.e., style and sportiness). Attributes within each dimension are interdependent as redundant measures of the same superordinate attribute (cf. Type II in Fig. 2). For example, riding comfort and roominess are perceived to share the same meaning (i.e., overall comfort). That is, people may form their judgement about overall comfort by abstracting two pieces of information, namely: riding

A. Representation (For simplicity, measurement errors are not shown.)

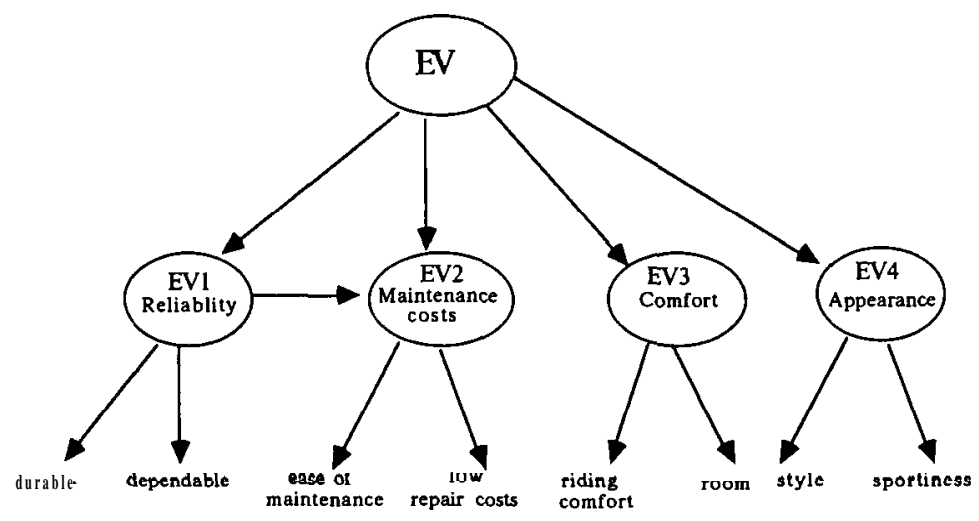

B. Specification

$\left(\begin{array}{l}Y_{1} \\ Y_{2} \\ Y_{3} \\ Y_{4} \\ Y_{5} \\ Y_{6} \\ Y_{7} \\ Y_{8}\end{array}\right)=\left(\begin{array}{cccc}\lambda_{1} & 0 & 0 & 0 \\ \lambda_{2} & 0 & 0 & 0 \\ 0 & \lambda_{3} & 0 & 0 \\ O h 40 & 0 \\ 0 & 0 h 5 & 0 \\ 0 & 0 & \lambda_{6} & 0 \\ 0 & 0 & 0 & \lambda_{7} \\ 0 & 0 & 0 & \lambda_{8}\end{array}\right)+\left(\begin{array}{l}\eta_{1} \\ \eta_{2} \\ \eta_{3} \\ \eta_{4}\end{array}\right)+\left(\begin{array}{l}\varepsilon_{1} \\ \varepsilon_{2} \\ \varepsilon_{3} \\ \varepsilon_{4} \\ \varepsilon_{5} \\ \varepsilon_{6} \\ \varepsilon_{7} \\ \varepsilon_{8}\end{array}\right)$

$\left(\begin{array}{l}\eta_{1} \\ \eta_{2} \\ \eta_{3} \\ \eta_{4}\end{array}\right)=\left(\begin{array}{llll}0 & 0 & 0 & 0 \\ \beta & 0 & 0 & 0 \\ 0 & 0 & 0 & 0 \\ 0 & 0 & 0 & 0\end{array}\right)\left(\begin{array}{l}\eta_{1} \\ \eta_{2} \\ \eta_{3} \\ \eta_{4}\end{array}\right)+\left(\begin{array}{l}\gamma_{1} \\ \gamma_{2} \\ \gamma_{3} \\ \gamma_{4}\end{array}\right) \xi+\left(\begin{array}{l}\zeta_{1} \\ \zeta_{2} \\ \zeta_{3} \\ \zeta_{4}\end{array}\right)$

where $\boldsymbol{\xi}$ is the interdependence $\mathrm{EV}$ attitude and $\boldsymbol{\eta}_{\mathbf{i}}$ is $\mathbf{E V}_{\mathbf{i}}$, for $\mathbf{i}=1$ to 4 .

Fig. 3. Representation and specification of the interdependence EV model. 
comfort and roominess. Furthermore, two dimensions (i.e., reliability and maintenance costs) are likely to interdependent. Since reliability is likely to induce low maintenance costs, people may change their beliefs about maintenance costs as a function of their judgement or knowledge about reliability.

The interdependence EV model examined herein is illustrated in Fig. 3A. The interdependence EV model was specified by a set of equations, which are given in Fig. 3B. Each expectancy-value ( $B_{i} \mathrm{a}_{i}$ ) measure, designated as $Y_{1}$ to $Y_{8}$, was a function of an underlying factor (subdimension) and a measurement error. No correlations among the measurement errors were specified in the model, since there were no strong theoretical reasons for them (cf. Fornell, 1983). The model was estimated by employing a modified version of higherorder confirmatory factor analysis. ${ }^{4}$ The overall EV attitude was represented as a second-order factor, whereas its dimensions $\left(\mathrm{EV}_{1}\right.$ to $\mathrm{EV}_{4}$ ) were represented as first-order factors. The hypothesized dimensional structure was confirmed by this analysis.

Hypothesis 1 concerns the convergent validity of the interdependence EV model. Convergent validity was assessed by checking whether the specified EV model provides a satisfactory fit to the observed data (cf. Bagozzi, 1983; Burnkrant and Page, 1988). Results showed that the interdependence EV model achieved convergent validity; $\chi^{2}(15)=$ 22.84, $p>0.09$. Other measures of the overall fit of the model also suggested that the model was satisfactory: the goodness-of-fit index = 0.92 , and the root mean square residual $=0.05$ (Bagozzi and Yi, 1988).

\footnotetext{
4 Note that a second-order factor has no direct measures but rather achieves meaning through first-order factors which do have direct measures. General discussion of a higher-order factor analysis can be found elsewhere (see, e.g., Gerbing and Anderson, 1984). The proposed EV model is similar to the second-order factor analysis model, but different in that first-order factors are allowed to be interdependent.
}

But is the convergent validity of the interdependence EV model higher than that of existing EV models? To answer this question, similar analyses were conducted with existing EV representations. Results suggested that existing $\mathrm{EV}$ models did not achieve convergent validity: $\quad \chi^{2}(20)=207.01, p<0.001$ (the modified unidimensional EV model); $\chi^{2}(16)$ $=40.46, p<0.001$ (the multidimensional $\mathrm{EV}$ model). 5

The above analysis suggested support for Hypothesis 1, but a more rigorous test was conducted with a hierarchical model comparison (Jbreskog and Sörbom, 1984). Since existing EV models are nested models of the interdependence EV model, a model comparison was made by a $\chi^{2}$ difference $\left(\chi_{d}^{2}\right)$ test. 6 First, the interdependence EV model was compared with the multidimensional EV model; the $\chi_{\mathbf{d}}^{2}$ was 17.90 with one degree of freedom, which was significant at the 0.001 level. Second, the interdependence EV model was compared with the modified EV model; the $\chi_{\mathrm{d}}^{2}$ was 184.21 with 5 degrees of freedom, which was also significant at the 0.001 level. Overall, the interdependence EV model achieved higher convergent validity than existing models.

Table 1 presents the reliabilities of individual EV items, composite reliabilities, and average variance extracted (AVE) (Bagozzi and Yi, 1988; Fornell and Larcker, 1981). Although some individual item reliabilities were low, all composite reliabilities were

5 An alternative model with formative indicators was also estimated; that is, the arrows relating factors and indicators were reversed in direction. But the goodness-of-fit results were not satisfactory: $\chi^{2}(15)=529.64, p<0.001$.

6 This can be illustrated with the interdependence EV model in Fig. 3. If one constrains the causal path between $\mathbf{E V}_{1}$ and $\mathrm{EV}_{2}$ to zero in the interdependence $\mathrm{EV}$ model, it becomes a multidimensional model. A modified unidimensional model occurs with this zero constraint between $\mathrm{EV}$, and $\mathrm{EV}_{2}$ plus equality constraints for the loadings from a second-order factor $(\mathrm{EV})$ to first-order factors $\left(\mathrm{EV}_{i}\right.$ 's). Hence, the modified unidimensional, and multidimensional EV models are nested models of the interdependence EV model. 
Table 1

Factor loadings and reliabilities for the expectancy-value (EV) measures

\begin{tabular}{|c|c|c|c|c|}
\hline$\overline{\mathrm{EV} \text { item }}$ & $\begin{array}{l}\text { Standardized } \\
\text { factor loading }\end{array}$ & $\begin{array}{l}\text { Individual } \\
\text { item reliability }\end{array}$ & $\begin{array}{l}\text { Composite } \\
\text { reliability }\end{array}$ & $\begin{array}{l}\text { Average variance } \\
\text { extracted }\end{array}$ \\
\hline Highly durable & 0.90 & 0.75 & 0.80 & 0.77 \\
\hline Highly dependable & 0.81 & 0.59 & & \\
\hline Easy to maintain & 0.88 & 0.61 & 0.73 & 0.57 \\
\hline Requires low repair costs & 0.84 & 0.53 & & \\
\hline Provides riding comfort & 0.92 & 0.63 & 0.60 & 0.57 \\
\hline Provides enough room & 0.78 & 0.30 & & \\
\hline Has an appealing style & 0.97 & 0.78 & 0.68 & 0.67 \\
\hline Has a sporty appearance & 0.67 & 0.30 & & \\
\hline
\end{tabular}

higher than 0.60 , the usual cut-off level, and the average composite reliability is 0.71 . All the AVE measures were greater than 0.5, which is considered adequate (Fornell and Larcker, 1981). The average AVE was 0.61, suggesting that approximately more than $60 \%$ of variance in the constructs were accounted for by the measures. All factor loadings exceeded 0.5 , a level considered high by researchers (see, e.g., Green, 1978). Overall, the measures of EV attitude were reliable.

\subsection{Implications of EV attitude representation}

\section{Effects of EV attitude on intentions}

Hypothesis 2 predicts that representations of EV attitude will influence the estimate of the direct effect of $\mathrm{EV}$ attitude on intentions (BI); that is, the direct effect will be underestimated under the traditional EV model. This prediction was tested by comparing the direct effect estimates observed under alternative EV models. In assessing the effects of EV attitude on intentions, subjective norm was included as another antecedent (Ajzen and Fishbein, 1980). Table 2 summarizes the findings.

First, the effects of EV attitude on BI were examined under the interdependence EV model. The direct effect of EV attitude on BI was 0.16 , which was significant at the 0.05 level. Consistent with the usual prediction, EV attitude had significant indirect effects on $\mathrm{BI}$ via A-act; $\mathrm{EV}$ attitude had significant ef- fects $(0.32$ with $t=3.0)$ on A-act which in turn had significant effects $(0.28$ with $t=3.6)$ on BI.

Next, the effects of EV attitude were examined under the traditional EV model. The direct path from EV attitude to BI was significant $(0.17$ with $t=2.2)$. The indirect effects were also significant, with $0.27(t=3.5)$ for the path from $\mathrm{EV}$ attitude to A-act and $0.46(t=5.1)$ for the path from A-act to BI. It can be noted that the parameter estimates are quite similar between the traditional and interdependence EV models (e.g., 0.16 vs. 0.17 from $\mathrm{EV}$ to $\mathrm{BI}, 0.32$ vs. 0.34 from $\mathrm{EV}$ to A-act, and 0.28 vs. 0.27 from $\mathrm{A}$-act to $\mathrm{BI}$, respectively).

Similar analyses were done with the modified unidimensional EV model and the multidimensional EV model. As Table 2 shows, all the direct effects were quite similar in their magnitude and significance under different EV models; different representations of $\mathrm{EV}$ attitude did not lead to different conclusions

Table 2

Effects of EV attitude under different models

\begin{tabular}{llllll}
\hline EV model & \multicolumn{2}{l}{ Direct } & effect & & Indirect effect \\
\cline { 2 - 3 } \cline { 5 - 6 } & EV $\rightarrow$ BI & & EV $\rightarrow$ A-act & A-act $\rightarrow$ BI \\
\hline Int-EV & $0.16^{\mathbf{a}}(1.8)$ & $b$ & $0.32(3.0)$ & $0.28(3.6)$ \\
Tra-EV & 0.17 & $(2.2)$ & & $0.34(3.7)$ & $0.27(3.5)$ \\
Mod-EV & 0.18 & $(2.1)$ & & $0.42(3.9)$ & $0.25(3.1)$ \\
Mul-EV & 0.18 & $(1.9)$ & & $0.44(4.1)$ & $0.25(3.0)$ \\
\hline
\end{tabular}

a These are standardized parameter estimates.

b Critical ratios are within parentheses. 
as to the direct effects of EV attitude. The effect of subjective norm on BI was also invariant across representations of $\mathrm{EV}$ attitude, ranging from 0.51 to 0.53 . In sum, both direct and indirect effects of EV attitude are invariant under alternative EV models, and Hypothesis 2 is not supported.

\section{Belief change}

Prior to testing Hypothesis 3, it was necessary to identify interdependent beliefs. The standardized estimate for the interdependence link between $\mathrm{EV}_{1}$ (reliability) and $\mathrm{EV}_{2}$ (low maintenance costs) dimensions was 0.54 $(t=4.73, \quad p<0.001)$, suggesting that reliability is causally associated with low maintenance costs as hypothesized in the interdependence EV model. As a result, the four attributes (i.e., dependability, durability, ease of maintenance, and low repair costs) measuring these two dimensions were found to be interdependent (see Fig. 3A). Other possible dependencies among dimensions (e.g., style and comfort) were analyzed, but none of them were statistically significant.

The implications of the network-like EV representation for belief change were tested by examining the effects of a persuasive attempt on interdependent beliefs. The belief change scores were calculated by the postminus pre-exposure beliefs for all attributes (A $B_{i}=B_{i}^{t_{2}}-B_{i}^{t_{1}}$, where $t_{1}$ and $t_{2}$ indicate pre- and post-exposure occasions, respectively). The results are summarized in Table 3. Recall that subjects saw one of the two types of advertisements; half of the subjects ( $n=60)$ saw the ad emphasizing high dependability, whereas the other half $(n=60)$ saw the ad claiming low repair costs.

First, belief changes were examined for the group that saw the ad mentioning dependability. Subjects' beliefs about the mentioned attribute (dependability) were changed significantly after ad exposure $\left(\Delta B_{i}=25, p<\right.$ $0.01)$. In addition, there were also significant
Table 3

Comparison of belief change scores $\left(\Delta B_{i}\right)$ after ad exposure

\begin{tabular}{lcc}
\hline Belief & \multicolumn{2}{l}{$\begin{array}{l}\left.\text { Belief change scores ( A } B_{i}\right) \\
\text { after ad exposure a }\end{array}$} \\
\cline { 3 - 4 } & $\begin{array}{l}\text { Ads claiming } \\
\text { high depend- } \\
\text { ability } \\
(n=60)\end{array}$ & $\begin{array}{l}\text { Ads claiming } \\
\text { low repair } \\
\text { costs } \\
(n=60)\end{array}$ \\
\hline Highly durable & $1.28^{\mathrm{b}}$ & $0.80^{\mathrm{b}}$ \\
Highly dependable & $1.25^{\mathrm{b}}$ & $0.63 \mathrm{~b}$ \\
Easy to maintain & $0.68^{\mathrm{b}}$ & $0.78^{\mathrm{b}}$ \\
Requires low repair costs & $1.35^{\mathrm{b}}$ & $1.58^{\mathrm{b}}$ \\
Provides riding comfort & 0.17 & -0.38 \\
Provides enough room & $0.48^{\mathrm{b}}$ & 0.20 \\
Has an appealing style & $\mathbf{- 0 . 4 5}$ & -0.17 \\
Has asporty appearance & -0.12 & 0.23
\end{tabular}

a Post-exposure beliefs minus pre-exposure beliefs.

${ }^{b} p<0.01$

c $p<0.05$.

changes in the subjects' beliefs about other attributes (i.e., durability, ease of maintenance, and low repair costs) which were interdependent with the mentioned attribute. Specifically, the belief changes were 1.28 (durability), 0.68 (case of maintenance costs), and 1.35 (low repair costs), which were all significant at the 0.01 level. There was an unexpected finding as well; there were changes in subjects' beliefs about roominess which was neither mentioned nor interdependent with the mentioned attribute (A $B_{i}=0.48$, $p<0.05)$. This unexpected finding might be explained by a halo effect (Holbrook, 1983).

Belief change scores were also assessed for the group that saw ads emphasizing low repair costs. The belief changes for the four interdependent attribute were all significant at the 0.01 level. Specifically, the belief change scores were 0.80 (durability), 0.63 (dependability), 0.78 (ease of maintenance costs), and 1.58 (low repair costs). Overall, Hypothesis 3 is supported; an ad designed to change a belief influenced clusters of interconnectedbeliefs. 


\section{Discussion}

The findings of this study show that $\mathrm{EV}$ attitude can be represented in a network form of interrelated elements. Evidence suggests that the interdependence EV model is a valid representation of EV attitude. The interdependence EV model retains information about the structure of EV attitude, rather than collapsing it into a single summary value as has been done in the past (Ajzen and Fishbein, 1980). This model is consistent with the associative network model of memory by describing the associations among EV elements as interdependencies (Anderson, 1983; Anderson and Bower, 1973), but it makes different types of associations explicit, whereas the associative network model does not.

This representation supports and broadens the recent findings that EV attitude has a hierarchical structure with subdimensions (Oliver and Bearden, 1985; Shimp and Kavas, 1984). The interdependence EV model extends such findings by delineating horizontal as well as vertical structure of EV attitude; vertical structure is represented with superordinate and subordinate attributes, whereas horizontal structure is represented with the interdependence linkages among attitudinal elements at the same level of abstraction.

This study examined whether representations of EV attitude affect the observed effect of EV attitude on intentions (BI). EV attitude had both direct and indirect effects on BI, but the effects did not vary across different representations. Even when EV attitude was represented as a single summary value, estimates of and conclusions about the effects of EV attitude on BI were not affected. This finding fails to support what researchers predicted as the consequence of misrepresenting multidimensional constructs (Bagozzi, 1983). That is, treating measures of multidimensional EV attitude as unidimensional did not lead to different predictions of its effects than otherwise.

Past research specifies that EV attitude influences BI through its effect on A-act (Fishbein and Ajzen, 1975; Ajzen and Fishbein, 1980; Lutz, 1977). But are the effects of EV on BI all mediated by A-act? This question has been raised as one of the challenges to the so-called sufficiency of the Fishbein model (Bettman, 1986). The present study shows that EV attitude can have not only indirect effects on BI through A-act, but also direct effects unmediated by A-act. This finding suggests that A-act is not sufficient to predict $\mathrm{BI}$ and that one should include EV attitude to predict BI accurately.

A persuasive communication indirectly influenced other unmentioned beliefs which were connected with the mentioned belief. This finding fits well with our representation of EV attitude. Since EV attitude exists as a cognitive network of interconnected beliefs, an ad effect on one belief is expected to bring about changes in other related belief. This is also consistent with the spreading-activation theory (Collins and Loftus, 1975). That is, when consumers are exposed to an ad, clusters of interdependent beliefs change together.

This study extends past attitude theory in several respects. It deepens our understanding of attitude by identifying the substructures underlying EV attitude. The interdependence EV model delineates the relations among attributes and helps us understand how individual judgments and evaluations function to form an attitude. Also, the interdependence EV model is broad in that it can represent various structures of $\mathrm{EV}$ attitude.

By using the structure of EV attitude as a basis for understanding advertising effects, this study links attitude formation and attitude change. Recent studies of attitude formation have found the existence of substructures in EV attitude (Bagozzi, 1982; Oliver and Bearden, 1985; Shimp and Kavas, 1984), 
but its implications for attitude change have not been investigated. This study has shown that the attitude structure can provide diagnostic information for predicting advertising effects on belief change.

The present study has several practical implications as well. This research may help practitioners to identify potentially dysfunctional second-order effects. For example, when the Pringles potato chips were introduced in a can, the initial enthusiasm of some consumers turned negative. Allegedly, consumers developed an unfavorable attitude toward the taste of Pringles (which prior research had indicated tasted as good as regular chips) on the basis of beliefs of artificiality. Perceptions of taste were affected by secondorder influences arising from the 'perfect shape' of chips, can, and other non-taste attributes. Understanding interdependencies among product attributes will permit us to anticipate such second-order effects.

Advertisers might use an understanding of attribute interdependence in designing ad messages. In general, some beliefs are more difficult to change through external attempts than others. In such cases, it may be effective to focus on beliefs that are relatively easy to change yet are related to the intended beliefs. The ad will induce the change in the mentioned (more vulnerable) beliefs, which will in turn induce changes in the originally intended beliefs. Thus, the structure of EV attitude can be used to identify which attributes of a product should be attacked in ad campaigns.

This study also gives useful insights for maximizing communication effects with minimum repetition. For example, if consumers perceive that several attributes (riding comfort and safety) are caused by a common antecedent (the size of a car), then concentrating ads on that antecedent may be more efficient in early stages of a campaign than repeating messages about each' attribute. Later, as wearout occurs, the advertiser can shift its focus to one or more of the second- order attributes.

Several caveats of this study and directions for future research are in order. This study used only one method (i.e., self-report) to measure key concepts. Future replication of the study might use multiple methods to eliminate the rival explanation of shared method variance. This study was conducted in high involvement situations (with print ads for an automobile), and the subjects (from the West Coast of the United States) were restricted in nature. Future research should test whether the findings are generalizable to other situations.

One can further explore the following questions concerning the structure of $\mathrm{EV}$ attitude. Under what conditions does the interdependent structure occur for EV attitude? We have found an interdependent structure in this study, but it is unlikely that EV attitude has always an interdependent structure. For example, simpler representations such as the traditional EV model might constitute enough means for representing EV attitude toward some acts with a small number of consequences. 'What factors will facilitate the activation and utilization of the interdependence linkage by consumers? For instance, consumer expertise might increase the perception of interdependence between product attributes. Which type of interdependence is more important than others? For example, is a causal relation more accessible to consumers than a semantic relation? This study showed that one benefit of representing $\mathrm{EV}$ attitude as a structure is diagnostic information for dynamics of belief change. But what other benefits exist? Probing answers to these questions will enrich our understanding of attitude formation and change processes.

\section{Acknowledgment}

The author thanks the editor, Professor Gilles Laurent, three anonymous reviewers, Richard Bagozzi, and Johann Baumgartner 
for their helpful comments on an earlier version of this paper.

\section{References}

Ajzen, I. and M. Fishbein, 1980. Understanding attitudes and predicting social behavior. Englewood Cliffs, NJ: PrenticeHall.

Anderson, J.R., 1983. The architecture of cognition. Cambridge, MA: Harvard University Press.

Anderson, J.R. and C.H. Bower, 1973. Human associative memory. Washington, D.C.: Winston \& Sons.

Bagozzi, R.P., 1981. An examination of the validity of two models of attitude. Multivariate Behavioral Research 16, 323-359.

Bagozzi, R.P., 1982. A field investigation of causal relationships among cognitions, affect, intentions, and behavior. Journal of Marketing Research 19, 562-584.

Bagozzi. R.P., 1983. A holistic methodology for modeling consumer response to innovation. Operation Research 31, 128-176.

Bagozzi, R.P., 1985. Expectancy-value attitude models: An analysis of critical theoretical issues. International Journal of Research in Marketing 2, 43-60.

Bagozzi, R.P. and Y. Yi, 1988. On the evaluation of structural equation models. Journal of the Academy of Marketing Science 16, 74-94.

Beckwith, N.E., and D.R. Lehmann, 1973. The importance of differential weights in multiple attribute models of consumer attitude. Journal of Marketing Research 10, 141145.

Bettman, J.R., 1986. Consumer psychology. Annual Review of Psychology 37, 257- 289.

Burnkrant, R.E. and T.I. Page, 1988. The structure and antecedents of the normative and attitudinal components of Fishbein's theory of reasoned action. Journal of Experimental Social Psychology 24, 66-87.

Collins, A.A. and E.F. Loftus, 1975. A spreading activation theory of semantic processing. Psychological Review 82, $407-428$
Fishbein, M. and I. Ajzen, 1975. Belief, attitude, intention and behavior: An introduction to theory and research. Reading, MA: Addison-Wesley.

Fornell, C., 1983. Issues in the application of covariance structure analysis. Journal of Consumer Research 9, 443-448.

Fornell, C. and D.F. Larcker, 1981. Evaluating structural equation models with unobservable variables and measurement errors. Journal of Marketing Research 18, 39- 50.

Gerbing, D.W. and J.C. Anderson, 1984. On the meaning of within-construct correlated errors. Journal of Consumer Research 11, 572-580.

Green, P.E., 1978. Analyzing multivariate data. Hinsdale, IL: Dryden Press.

Holbrook, M.B., 1983. Using a structural equation model of halo effect to assess perceptual distortion due to affective overtones. Journal of Consumer Research 10, 247-252.

Huber, J. and J. McCann, 1982. The impact of inferential beliefs on product evaluations. Journal of Marketing Research 19, 324-333.

Jöreskog, K.G. and D. Sörbom, 1984. LISREL VI: User's guide. Mooresville, IN: Scientific Software, Inc.

Liska, A., 1984. A critical examination of the causal structure of the Fishbein/Ajzen attitude-behavior model. Social Psychology Quarterly 47, 61-74.

Lutz, R.J., 1977. An experimental investigation of causal relationships among cognitions, affect, and behavioral intentions. Journal of Consumer Research 3, 197-208.

Oliver, R.L. and W.O. Bearden, 1985. Crossover effects in the theory of reasoned action: A moderating influence attempt. Journal of Consumer Research 12, 324-340.

Schlegel. R.P. and D. DiTecco, 1982. Attitudinal structures and the attitude-behavior relation. In: M.P. Zanna, E.T. Higgins and C.P. Herman (eds.), Consistency insocial behavior. Hillsdale, NJ: Erlbaum, 17-52.

Shimp, T.A. and A. Kavas, 1984. The theory of reasoned action applied to coupon usage. Journal of Consumer Research $11,795-809$.

Wilkie, W.L. and E.A. Pessemier, 1973. Issues in marketing's use of multi-attribute attitude models. Journal of Marketing Research 10. 428-441. 